\section{Les rayons, l'ADN et la flèche du temps}

Des horloges biologiques règlent chaque moment de la vie, égrénant les heures du jour et de la nuit... et marquant le temps qu'il reste à vivre. Chez la Drosophile et le champignon Neurospora crassa, les mécanismes moléculaires des systèmes oscillatoires réglant le rythme nycthéméral et son ajustement aux alternances jour/nuit commencent maintenant à être bien connus. Dans les deux cas, mais par des mécanismes différents, posttraductionnels dans le premier cas et transcriptionnels dans le second, la lumière semble l'élément déterminant de l'ajustement du système. L'horloge gouvernant la longévité des espèces est, quant à elle, encore mystérieuse; à vrai dire, les mécanismes en cause pourraient être très différents selon les types de cellules vivantes. Chez l'Homme, la découverte que le gène d'un syndrome génétique de vieillissement accéléré (le syndrome de Werner) code pour une hélicase renforce le sentiment qu'existent des relations étroites entre le vieillissement et la capacité de maintenir l'intégrité du génome, notamment contre les dommages causés par les radiations ionisantes diverses. Les ultra-violets sont bien connus pour causer un vieillissement accéléré de la peau. Dans les conditions épouvantables où persistent et se développent différentes formes de vie dans la région massivement contaminée de Tchernobyl, des résultats récents indiquent une augmentation extraordinaire des mutations mitochondriales chez les rongeurs, et une instabilité des minisatellite chez des personnes contaminées : outre les risques de cancers, cela aura-til une incidence sur la longévité moyenne? Des altérations de la longueur de répétitions de triplets peuvent-elles être engendrées par une irradiation externe, transformant alors des allèles normaux de certains gènes en allèles à risque d'amplification dans les générations futures? Comme on le voit, beaucoup de questions que l'on se serait bien passé de poser... mais auxquelles il pourrait être maintenant possible de répondre.

\title{
Mécanismes moléculaires du fonctionnement et de la remise à I'heure de I'horloge biologique
}

Chez tous les organismes vivants un grand nombre de comportements et de fonctions physiologiques sont exprimés de façon cyclique avec une période d'environ $24 \mathrm{~h}$ d'où le nom de rythme circadien donné à ces cycles. Ces oscillations sont normalement entraînées par des périodes jour-nuit (JN12:12) mais persistent aussi dans des conditions où l'alternance jour-nuit est supprimée, avec une période proche de $24 \mathrm{~h}$. Il existe donc chez tous les êtres vivants un système oscillatoire biologique capable de mesurer le temps, c'est-àdire une véritable horloge biologique. Cette horloge biologique doit être capable de communiquer l'information temps à tout l'organisme et doit aussi pouvoir être remise à l'heure en fonction de stimuli extérieurs. Quel est le mécanisme moléculaire d'une horloge biologique? Comment se fait la remise à l'heure de cette horloge? Comment cette heure biologique est-elle transmise aux différentes fonctions de l'organisme? C'est aux deux premières de ces questions que plusieurs équipes de chercheurs américains viennent de trouver des réponses en s'intéressant aux rythmes biologiques chez la drosophile.

period, le premier composant identifié de l'horloge biologique de la drosophile

C'est au début des années 1970 qu'un premier gène affectant le rythme circadien a été identifié chez la drosophile [1], le gène period (per) $\left(\mathrm{m} / \mathrm{s} n^{\circ} 4\right.$, vol. $2, p$. 223). Le clonage de ce gène a permis de montrer que le taux de l'ARN per oscillait cycliquement avec une période de $24 \mathrm{~h}$ il atteint son maximum vers $15 \mathrm{~h}$ et est à son minimum de 0 à $4 \mathrm{~h}$. Les conséquences phénotypiques de différents allèles mutés suggéraient fortement que le gène per contrôlait directement les rythmes biologiques: une perte de fonction abolit tout rythme circadien alors que d'autres allèles allongent ou raccourcissent la période des cycles. Le gène per code pour une protéine nucléaire qui règle négativement la transcription de son propre gène [2]. L'augmentation de la quantité d'ARN per entraîne la synthèse de protéine PER qui est localisée au noyau où elle réprime la transcription du gène per. Cela entraîne la diminution du taux des ARNm per et, par voie de conséquence, de la protéine PER qui, en repassant sous un certain seuil, permet la réactivation de la transcription du gène per. Le gène per code donc pour un élément essentiel de l'horloge biologique. Cependant, cette boucle d'autorégulation négative n'est pas suffisante pour obtenir une horloge perpétuelle. En effet ce seul mécanisme aboutirait rapidement à un amortissement de l'expression du gène considéré. Il faut ainsi envisager l'existence d'autres composants contribuant à retarder l'auto-inhibition de per pour permettre d'entretenir le mouvement de l'horloge biologique.

timeless code pour un deuxième composant de l'horloge biologique de la drosophile

L'isolement d'un deuxième mutant arythmique timeless (tim) chez la drosophile [3] a permis d'identifier un bon candidat pour être un partenaire essentiel de l'horloge biologique. En effet dans des mutants avec perte de fonction tim, l'expression ryth- 
mique de l'ARN per est abolie. C'est, chose inhabituelle chez la drosophile, par clonage positionnel [4] que le gène timeless a été cloné puis séquencé. Ce gène ne présente pas de domaine connu ni d'homologie avec le gène per. Le taux des messagers tim oscille sur une période de $24 \mathrm{~h}$ avec un maximum à la fin de la journée/début de nuit et un minimum à l'aube, comme pour les ARN per. En absence d'activité PER, les oscillations de l'ARN tim sont supprimées. La fonction TIM est requise pour assurer des oscillations régulières du taux de l'ARNm tim, indiquant une autorégulation de tim [5], comme nous venons de le voir pour le gène per. En absence d'activité TIM, les oscillations de l'ARN per sont aussi supprimées. Donc, les produits de ces deux gènes règlent négativement leur propre expression et sont nécessaires pour régler l'expression de l'autre gène, ce qui suggérait que ces deux gènes étaient bien au cœur du moteur de l'horloge biologique et que leurs produits devaient interagir. C'est en effet ce qui vient d'être démontré [6]. En recherchant des gènes codant pour des facteurs qui interagissent avec la protéine PER par le système des double hybrides chez la levure [6] on a trouvé que 16 clones sur les 48 isolés codaient pour la protéine TIM. L'analyse de ces clones a, de plus, permis de définir que la région 505-906 de la protéine TIM était suffisante pour l'interaction avec la protéine PER et que la région PER 233390 était cruciale pour cette interaction hétérophile. Les protéines PER et TIM sont donc des partenaires coopérant au fonctionnement de l'horloge biologique.

\section{Le retard à l'entrée de la protéine} PER dans le noyau assure

le maintien du fonctionnement de l'horloge

Il existe un retard important dans l'accumulation de la protéine PER puisqu'elle ne devient détectable que plus de $6 \mathrm{~h}$ après le début de l'accumulation de son ARN. De plus, la protéine PER commence à s'accumuler pendant la première heure dans la région périnucléaire avant

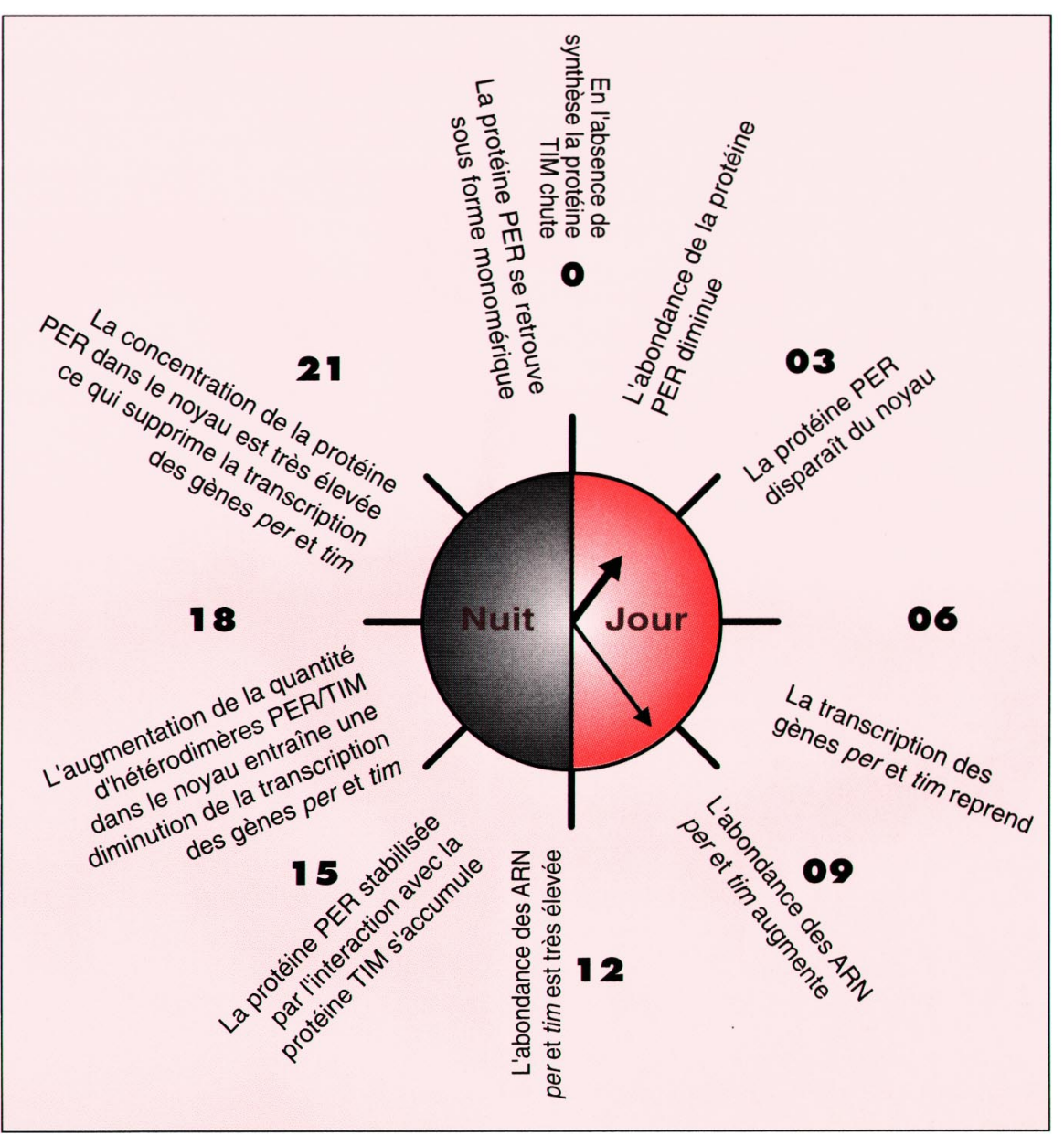

Figure 1. Les 24 heures d'une horloge biologique. Les principaux événements moléculaires décrits à ce jour, qui permettent l'oscillation continue de l'horloge biologique de la drosophile sont indiqués au moment où ils ont lieu pendant une période de 24 heures, dans des conditions d'éclairement $12 \mathrm{~h}$ de jour, $12 \mathrm{~h}$ de nuit (JN12:12). Les chiffres en gras indiquent les heures. En l'absence de lumière (24 $h$ de nuit), le cycle se maintient. La lumière permet d'avancer le cycle si l'éclairement a lieu entre 21 h et 24 h, et de le retarder s'il a lieu entre $12 \mathrm{~h}$ et $15 \mathrm{~h}$.

d'être transportée dans le noyau. La protéine PER réglant négativement la transcription de son gène, ces délais sont nécessaires pour le maintien de l'amplitude des oscillations de la transcription du gène per. Dans les mutants avec perte de fonction tim, la quantité de protéine PER est fortement diminuée, elle ne varie pas au cours de la journée et la protéine PER n'est pas transportée dans le noyau. La protéine PER n'est donc stable qu'en présence de protéine TIM. La formation d'hétérodimères PER/TIM serait dépendante de la concentration des deux partenaires [6]. Quand les messagers tim et per commencent à s'accumuler, ils ne seraient d'abord pas en quantité suffisante pour assurer la synthèse de suffisamment de protéine PER et TIM pour permettre la formation d'hétérodimères PER/TIM; la protéine PER ne serait ainsi pas transportée dans le noyau et serait même probablement détruite du fait de sa localisation cytoplasmique. La diminution de la quantité d'ARN per dans des mutants per entraîne, comme le prévoit ce modèle, un allongement de la période du cycle. Des mutants per diminuant la liaison de la protéine PER à la protéine TIM, caractérisée par la technique des double 
hybrides, entraînent également un retard à l'entrée de la protéine PER dans le noyau et allongent ainsi la période du cycle. Des expériences biochimiques récentes [7] ont montré que, chez la mouche, la protéine PER fait partie d'un complexe de haut poids moléculaire durant la période $10-20 \mathrm{~h}$. Ces complexes n'existent pas dans les mutants tim avec perte de fonction. En utilisant des protéines PER marquées, les auteurs [7] se sont aperçu que, quoique la protéine PER possède un domaine d'interaction protéine-protéine et soit capable de former des homodimères, il n'y avait pas d'homodimères PER dans ces complexes mais, à la place, des hétérodimères PER-TIM. Ces expériences confirment les résultats obtenus in vitro et montrent clairement que, chez la mouche, la majorité de la protéine PER forme un hétérodimère avec la protéine TIM pendant la période $10-20 \mathrm{~h}$ alors que pendant la période 1-4 h elle est essentiellement sous forme monomérique; les deux formes coexistent entre ces deux périodes.

\section{La protéine TIM est une clé essentielle pour la mise à l'heure de l'horloge}

En fin de nuit la diminution de la quantité de protéine TIM est très rapide et précède la diminution plus lente du taux de protéine PER [7], suggérant que le premier phénomène pourrait être la cause du second. La dégradation de la protéine TIM a donc été analysée en détail. Elle est plus rapide en condition jour-nuit qu'en condition nuit-nuit, suggérant un effet de la lumière sur sa dégradation.

Des mouches ont été exposées à la lumière à différents moments pendant la nuit. Dans tous les cas, cela a entraîné la dégradation rapide (en moins d'une heure) de la protéine TIM (mais pas de l'ARN tim), entrâ̂nant à son tour la production de monomères PER et leur dégradation lente $[7,8]$. Dans des mutants per, le gène tim s'exprime et la quantité de protéine TIM oscille; elle est faible pendant le jour et augmente pen- produisent que s'il y a alternance de jour et de nuit (JN12:12) mais pas si les mouches sont maintenues dans l'obscurité. Or chez ces mutants, le taux des transcrits tim ne change pas; donc, l'oscillation de l'abondance de la protéine TIM ne peut être due qu'à un mécanisme post-transcriptionnel tel que la dégradation de la protéine TIM induite par la lumière. La protéine TIM apparaît donc être une cible précoce de la lumière pour permettre la mise à l'heure de l'horloge biologique. En effet, en début de nuit, la lumière induit une dégradation de la protéine TIM qui est néanmoins rapidement remplacée car l'ARNm tim est abondant. Par conséquent, la lumière retarde le moment où un niveau suffisant de protéine TIM est atteint pour interagir avec la protéine PER, et retarde ainsi l'horloge biologique. A la fin de la nuit, en revanche, la lumière induit la dégradation de la majorité de la protéine TIM qui ne peut être remplacée car la transcription de son gène et le taux de son messager sont faibles, ce qui entraîne un relargage précoce de la protéine PER et donc avance l'horloge. C'est donc par un mécanisme de régulation post-transcriptionnelle que se fait la mise à l'heure de l'horloge biologique chez la drosophile. Afin de comprendre parfaitement cette boucle de régulation il reste à savoir si la lumière induit directement la dégradation de la protéine TIM ou, plus probablement, si elle active une protéase photosensible.

\section{Perspectives}

L'analyse des gènes per et tim a fait faire des progrès importants de la compréhension des mécanismes moléculaires qui assurent le fonctionnement et le réglage de l'horloge biologique de la drosophile en fonction des stimuli extérieurs. Il reste à montrer que ces mécanismes sont retrouvés chez d'autres organismes. Chez Neurospora crassa un gène frequency (frq) altérant les rythmes circadiens a été identifié. Il possède toutes les caractéristiques d'un gène codant pour un des composants de l'horloge biologique de ce champignon [9]. Une perte de la fonction du gène frq abolit la rythmicité, d'autres allèles changent la période des cycles. Le taux des transcrits frq suit un rythme circadien avec un maximum pendant le jour, donc à l'opposé de ce que l'on observe pour les transcrits per et tim chez la drosophile. La protéine FRQ règle négativement la transcription de son propre gène. De plus, un éclairement bref induit rapidement un pic d'expression de l'ARNm frq qui va entraîner un avancement du cycle si il a lieu en fin de nuit. Donc le gène frq, comme le produit du gène tim, est une cible primaire (ou un rouage très proche de la cible primaire) de la lumière. Une différence très importante est que la lumière règle la transcription du gène $f r q$ alors que, chez la drosophile, la mise à l'heure se fait par un mécanisme post-transcriptionnel. Il n'y a aucune homologie de séquence entre les gènes frq et tim, ce qui peut suggérer qu'il reste de nombreux composants des horloges biologiques à découvrir. Cependant, certains de ces composants ne sont peut-être pas spécifiques de cette fonction et pourraient être importants pour d'autres fonctions cellulaires, ce qui les rendrait alors difficile à identifier génétiquement.

Il reste par ailleurs de nombreuses autres boîtes noires dans la compréhension des mécanismes moléculaires des rythmes circadiens. Notamment comment le temps déterminé par l'horloge biologique est-il transmis aux effecteurs qui vont assurer les fonctions physiologiques et les comportements? Les gènes per, tim et frq règlent leur propre transcription; ils pourraient donc contrôler aussi la transcription de gènes codant pour ces effecteurs. Ces gènes pourraient être identifiés en recherchant des ARN dont le taux d'expression varie en fonction $\mathrm{du}$ rythme circadien mais dont la perte de fonction n'affecte pas le fonctionnement de l'horloge biologique. Les expériences effectuées chez la drosophile ont utilisé les têtes des mouches. Cependant, le gène per est exprimé de façon cyclique dans la plupart des tissus de l'adulte. Il ne semble donc pas y avoir, dans ce cas au moins, un centre spécialisé pour 
l'horloge biologique. Se pose alors le problème de la coordination entre les différentes cellules, mais aussi de la transmission des signaux extérieurs vers les différents organes. On ne sait cependant pas si les horloges biologiques qui pourraient exister dans différents organes du thorax et du corps fonctionnent de façon autonome ou requièrent des messages venus du système nerveux

\section{RÉFÉRENCES}

1. Konopka RJ, Benzer S. Clock mutants of Drosophila melanogaster. Proc Natl Acad Sci USA 1971 ; 68 : 2112-6.

2. Hardin PE, Hall JC, Rosbach M. Circadian oscillations in period gene mRNA levels are transcriptionally regulated. Proc Natl Acad Sci USA 1992; 89: 11711-5.

3. Sehgal A, Price JL, Man B, Young MW. Loss of circadian behavioral rhythms and per RNA oscillations in the Drosophila mutant timeless. Science 1994; 263: 1603-6.

4. Myers MP, Wager-Smith K, Wesley CS, Young MW, Sehgal A. Positional cloning and sequence analysis of the Drosophila clock gene, timeless. Science 1995; 270: 805-8.

5. Sehgal A, Rothenfluh-Hilfiker A, HunterEnsor M, Chen Y, Myers MP, Young MW. Rhythmic expression of timeless: a basis for promoting circadian cycles in period gene autoregulation. Science 1995; 270: 808-10.

6. Gekakis N, Saez L, Delahave-Brown, Myers MP, Sehgal A, Young MW, Weitz CJ. Isolation of timeless by PER protein interaction: defective interaction between timeless protein and long-period mutant $\mathrm{PER}^{\mathrm{L}}$. Science 1995 ; 270 : 810-5.

7. Zeng H, Qian Z, Myers MP, Rosbach M. A light-entrainment mechanism for the Drosophila circadian clock. Nature 1996; 380 : 12935.

8. Hunter-Ensor M, Ousley A, Sehgal A. Regulation of the Drosophila protein Timeless suggests a mechanism for resetting the circadian clock by light. Cell 1996; 84: 677-685.

9. Crosthwaite SK, Loros JJ, Dunlap JC. Light-induces resetting of a circadian clock is mediated by a rapid increase in frequency transcript. Cell 1995; 81 : 1003-12.

\section{Jean-Louis Couderc}

Inserm U. 384, 28, place Henri-Dunant, BP 38, 63001 Clermont-Ferrand Cedex, France.

\section{TIRÉS À PART}

J.L. Couderc.

\section{BRÈVES}

L'atrophie dentato-rubro-pallido-luysienne (DRPLA) serait d'origine japonaise. Alors que le nombre des maladies par expansion de triplets va toujours croissant, les recherches s'orientent aussi désormais sur l'origine, la répartition selon les ethnies, et les mécanismes favorisant l'instabilité des séquences répétées. On étudie la taille des séquences dans les populations normales et on examine la distribution des polymorphismes autour ou dans la séquence et/ou le gène. L'atrophie dentato-rubropallido-luysienne (DRPLA), une des maladies par expansion de triplets CAG $\left(m / s n^{\circ} 4, v o l .10, p .472\right)$, est plus fréquente au Japon, ses manifestations cliniques y sont un peu différentes et l'anticipation* y est beaucoup plus marquée que dans les populations occidentales. C'est pourquoi plusieurs groupes japonais se sont associés à des équipes coréenne, danoise et américaine pour faire une étude comparative de la répartition des haplotypes chez les Orientaux, les Européens et les Afro-Américains [1]. Deux polymorphismes intragéniques bialléliques se sont révélés très utiles: $\mathrm{A}$, qui est une substitution d'un nucléotide dans l'intron 1 , et $\mathrm{B}$, substitution analogue dans l'intron 3 (figure 1). Tous les malades japonais ont l'haplotype A1B1 retrouvés aussi chez les malades chinois et co- réens. Dans les populations normales, on trouve plus fréquemment l'haplotype A1B1 chez les Japonais, souvent associé à une séquence longue (17 répétitions), A1B2 chez les Européens, et A2B2 chez les Africains. L'haplotype A2B1 n'est jamais observé. En revanche, dans les familles atteintes de DRPLA européenne (danoise) et nord-américaine on a retrouvé l'haplotype A1B1, ce qui suggère que le DRPLA serait d'origine très ancienne et japonaise, bien que les familles caucasiennes testées n'aient pas d'ascendant asiatique connu. D'après les analyses faites chez les rongeurs et les primates, l'haplotype A2B2 serait la forme ancestrale. Les auteurs tirent donc de leurs constatations les conclusions suivantes: la mutation A1 est survenue la première, puis a été suivie de la mutation B1. Elles seraient apparues au Japon et seraient corrélées à une expansion des triplets CAG. L'haplotype A1B1, rare chez les Européens, serait venu des Japonais, introduit par un mélange de populations, peut-être au temps des grandes invasions de l'empire mongol. On attend maintenant de connaître la distribution des haplotypes chez les Indiens d'Amérique, puisqu'ils sont considérés comme des descendants des Mongols.

[1. Yanagisawa $\mathrm{H}$, et al. Hum Mol Genet 1996; 5 : 373-9.]

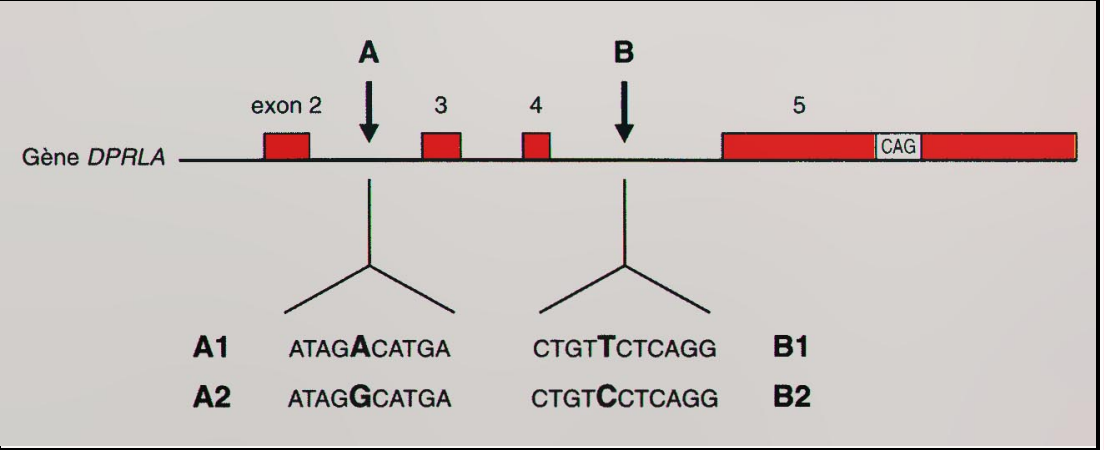

Figure 1. Gène DRPLA. I/ existe deux polymorphismes intragéniques dans les introns 1 et 3 qui déterminent la possibilité de 4 haplotypes: A1B1, le plus fréquent chez les Japonais et toujours retrouvé chez les malades, quelle que soit leur origine géographique, A1B2 chez les Européens et A2B2 chez les Africains. L'haplotype A2B1 n'a jamais été trouvé. 